-Supporting Information

\title{
Light Driving and Monitoring Growth of Single Gold Nanorods
}

Yingbo He, ${ }^{[1]}$ Yuqing Cheng, ${ }^{[1]}$ Jingyi Zhao, ${ }^{[1]}$ Xin-Zheng Li, ${ }^{[1]}$ Qihuang Gong, ${ }^{[1,2]}$ and Guowei $\mathrm{Lu}^{\left[1,2,{ }^{*}\right]}$

[1] State Key Laboratory for Mesoscopic Physics \& Collaborative Innovation Center of Quantum Matter, Department of Physics, Peking University, Beijing 100871, China

[2] Collaborative Innovation Center of Extreme Optics, Shanxi University, Taiyuan, Shanxi 030006, China.

E-mail: guowei.lu@pku.edu.cn 
(a)

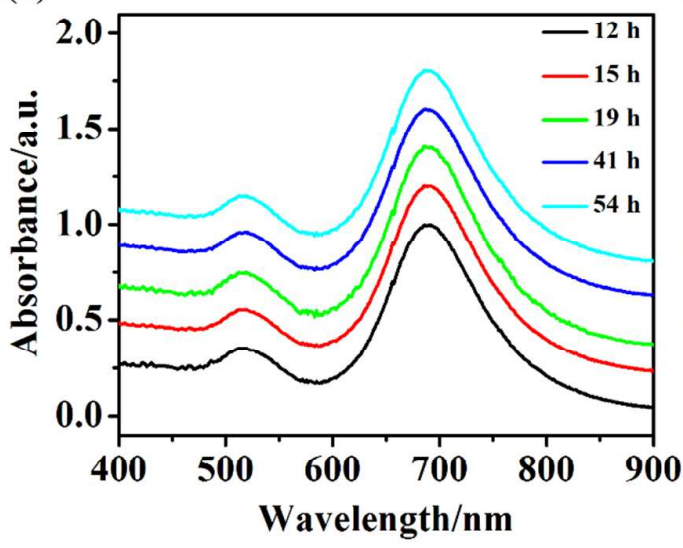

(b)

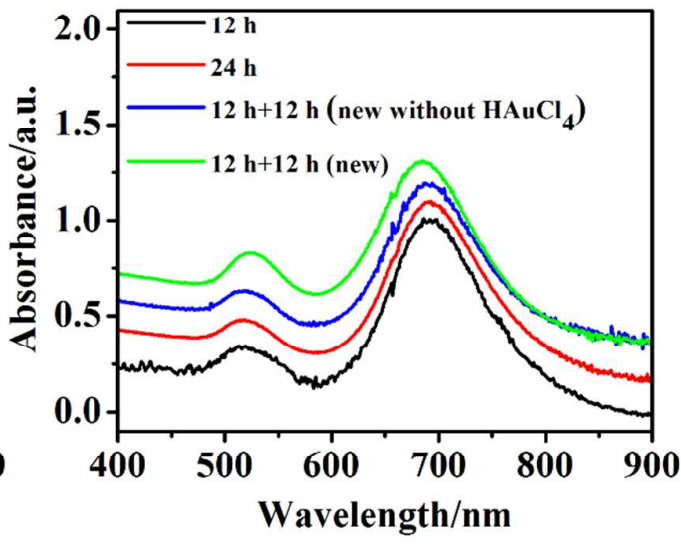

Figure S1. (a) The extinction spectra of the AuNRs solution after seed solution added about $12 \mathrm{~h}, 15 \mathrm{~h}, 19 \mathrm{~h}, 41 \mathrm{~h}$, and $54 \mathrm{~h}$ later. After growing $12 \mathrm{~h}$, the extinction spectra of the AuNRs solution show no obvious change. That means the AuNRs have grown up to an equilibrium state in the growth solution. (b) After $12 \mathrm{~h}$ growth of the AuNRs in solution (the black line), the AuNRs was divided into three portions. The first part continued to grow $12 \mathrm{~h}$ in the original growth solution (the red line). The second part was centrifuged and added into the new fresh growth solution which is the same composition and concentration with the surface growth solution but without any $\mathrm{HAuCl}_{4}$ (the blue line). The third part was centrifuged and added into the new fresh growth solution which is the same composition and concentration with the surface growth solution (the green line). After $12 \mathrm{~h}$ growth, the spectrum (the green line) only showed a little blue shift ( $\sim 6 \mathrm{~nm})$, which was probably due to the AuNRs growth in the short axis direction more. 
(a)

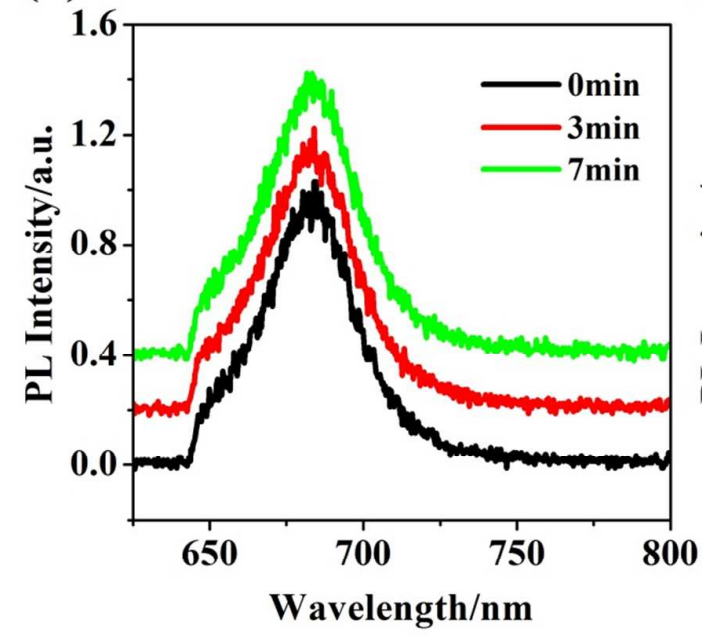

(b)

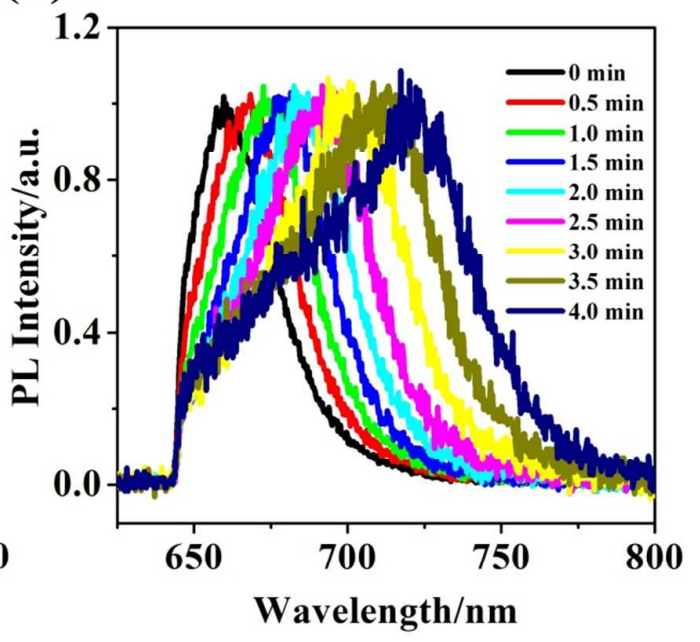

Figure S2. The influence of 633-nm laser illumination on the AuNRs growth. (a) The 633-nm laser was switched on only when the measurement was carried on. (b) The 633-nm laser was always switched on. Both (a) and (b) were measured on the same AuNR. 
(a)

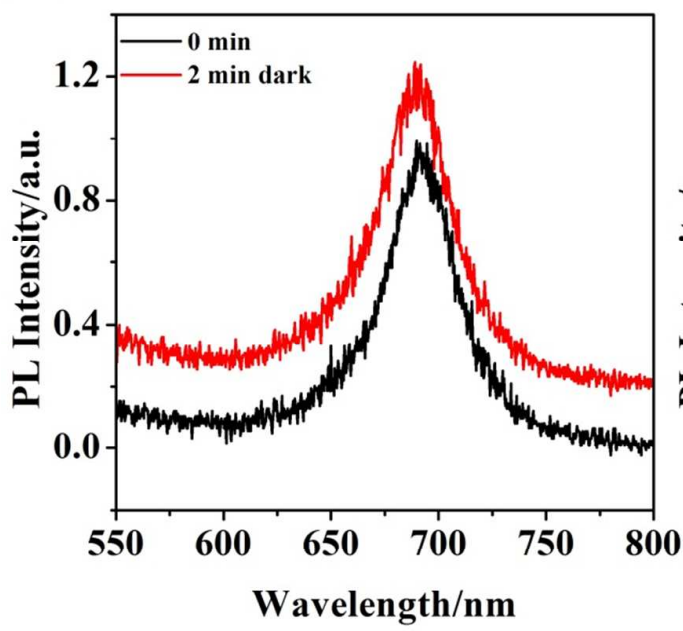

(c)

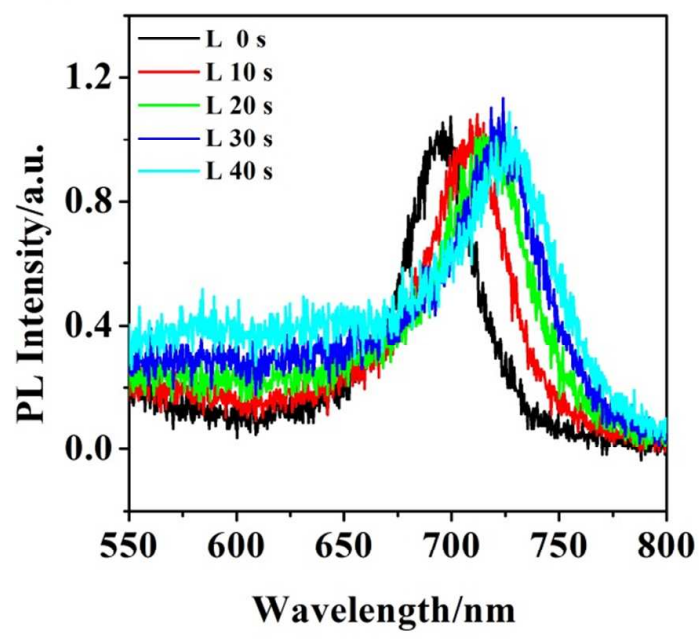

(b)

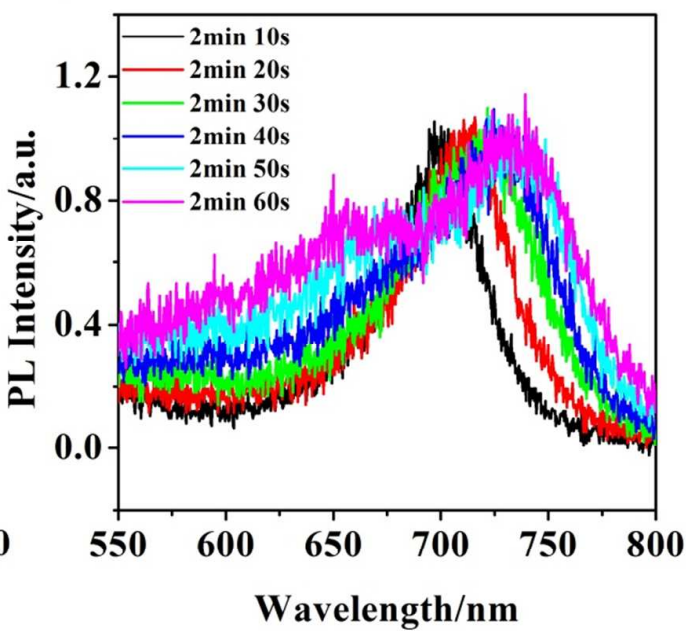

Figure S3. The influence of 532-nm laser illumination on the AuNRs growth. (a) In dark condition. (b) The laser was always on and the polarization was paralleled along the long axis of the AuNR. Both (a) and (b) were measured on the same AuNR. (c) The laser was always on and the polarization was perpendicular along the long axis of the AuNR. The laser power is about $100 \mu \mathrm{W}$. 